\title{
A randomised, double-blind, controlled trial comparing two intra-articular hyaluronic acid preparations differing by their molecular weight in symptomatic knee osteoarthritis
}

\author{
Francis Berenbaum, ${ }^{1}$ Joachim Grifka, ${ }^{2}$ Sara Cazzaniga, ${ }^{3}$ Massimo D'Amato, ${ }^{3}$ \\ Giampaolo Giacovelli, ${ }^{3}$ Xavier Chevalier, ${ }^{4}$ Francois Rannou, ${ }^{5}$ Lucio C Rovati, ${ }^{3}$ \\ Emmanuel Maheu ${ }^{1}$
}

\begin{abstract}
- Additional supplementary data are published online only. To view the files please visit the journal online (http://ard.bmj. com/content/early/recent).

${ }^{1}$ Department of Rheumatology, AP-HP Saint-Antoine Hospital, Paris, France

2Department of Orthopaedics, University Hospital, Regensburg, Germany

${ }^{3}$ Department of

Clinical Pharmacology,

Rottapharm |Madaus,

Monza, Italy

${ }^{4}$ Department of Rheumatology AP-HP Henri Mondor Hospital, University Paris XII,

Créteil, France

${ }^{5}$ Department of Rehabilitation, AP-HP Cochin Hospital, ParisDescartes University, Paris, France
\end{abstract}

\section{Correspondence to}

Emmanuel Maheu, AP-HP Saint-Antoine Hospital, 184 rue du faubourg Saint-Antoine, 75012 Paris, France;

emaheu@wanadoo.fr

Received 23 December 2011 Accepted 31 December 2011 Published Online First 31 January 2012

\section{ABSTRACT}

Objectives To compare the effects of an intermediate molecular weight (MW) intra-articular hyaluronic acid (HA) with a low MW product on knee osteoarthritis (OA) symptoms.

Methods Patients with symptomatic knee OA were enrolled inarandomised, controlled, double-blind, parallelgroup, non-inferiority trial with the possibility to shift to superiority. Patients were randomised to GO-ON(MW 800-1500 kD, 25 mg/2.5 ml) or Hyalgan(MW 500-730 $\mathrm{kD}, 20 \mathrm{mg} / 2 \mathrm{ml}$ ) injected at 3-weekly intervals. The primary outcome was 6-month change in the WOMAC pain subscale $(0-100 \mathrm{~mm})$. Sample size was calculated on a non-inferiority margin of $9 \mathrm{~mm}$, lower than the minimum perceptible clinical improvement. Secondary endpoints included OARSI-OMERACT responder rates Results The intention-to-treat (ITT) and per-protocol (PP) populations consisted of 217 and 209 patients and 171 and 172 patients in the GO-ON and Hyalgan groups, respectively. ITT WOMAC pain of $47.5 \pm 1.0$ (SE) and $48.8 \pm 1.0 \mathrm{~mm}$ decreased by $22.9 \pm 1.4 \mathrm{~mm}$ with $\mathrm{GO}-\mathrm{ON}$ and $18.4 \pm 1.5 \mathrm{~mm}$ with Hyalgan after 6 months. The primary analysis was conducted in the PP population followed by the ITT population.Mean (95\% CI) differences in WOMAC pain change were $5.2(0.9$ to 9.6$) \mathrm{mm}$ and 4.5 (0.5 to 8.5$) \mathrm{mm}$, respectively,favouring GO-ON, satisfying the claim for non-inferiority (lower limit $>-9 \mathrm{~mm}$ ) and for statistical superiority (95\% Cl all $>0, p=0.021)$. Ahigher proportion of OARSI/OMERACT responders was observed with G0-ONthan with Hyalgan (73.3\% vs58.4\%, $p=0.001)$. Both preparations were well tolerated.

Conclusions Treatment with 3-weekly injections of intermediate MW HA may be superior to low MW HA on knee OA symptoms over 6 months, with similar safety.

Hyaluronic acid (HA) is a glycosaminoglycan constituent of synovial fluid and cartilage matrix in normal joints. In osteoarthritis, HA molecular weight (MW) and concentration are decreased. Exogenous HA is available as a viscosupplementation device or a drug for intra-articular use in the treatment of knee osteoarthritis symptoms. ${ }^{1}$ Different HA formulations are currently available worldwide: from the reference low MW preparation (range 500 000-730000 Daltons) to more recent intermediate $\mathrm{MW}$ (range
800 000-2 000 000) and even cross-linked, high MW formulations (average 6000000 Daltons) including hylans, non-animal-derived HA and others. However, the efficacy of HA in knee osteoarthritis is still debated, which is reflected in several meta-analyses; ${ }^{2-7}$ the majority suggest small to strong effects of $\mathrm{HA},{ }^{2-5}$ while a minority failed to show a greater effect than placebo. ${ }^{6} 7$ Despite controversies, HA injections are recommended by the current Osteoarthritis Research Society International (OARSI) guidelines and previous practice guidelines. $^{8}$

While a more recent re-appraisal suggests that there is poor evidence of significant pain relief when the analysis is restricted to high-quality studies, ${ }^{9}$ one early meta-analysis ${ }^{4}$ suggested that the large heterogeneity between trials might be caused by the possible greater efficacy of high $\mathrm{MW}$ HA products. This was recently negated by a metaanalysis ${ }^{10}$ in which high MW, cross-linked HA was not significantly superior to lower MW preparations, but had a doubling frequency of post-injection joint flares. In addition, HA was found to have longer-lasting pain control compared with intra-articular corticosteroids, and the majority of trials has been performed with the low MW HA product. ${ }^{11}$

Low MW HA thus often remains the preferred option when using HA in knee osteoarthritis. However, there is a paucity of appropriately sized, high-quality trials comparing the effects of different MW preparations, with particular regard to potential differences between low and intermediate $\mathrm{MW}$ products, given the worse safety profile of high MW formulations. ${ }^{10}$ The present study was therefore designed to compare the effects of the reference low MW HA product (Hyalgan) with a well-characterised intermediate MW preparation (GO-ON) on knee osteoarthritis symptoms.

\section{METHODS \\ Study design}

This was a multicentre (50 orthopaedics and/ or rheumatology practice sites in France and Germany), prospective, randomised, double-blind, controlled, parallel-group trial, to compare the efficacy and safety of GO-ON with that of Hyalgan 


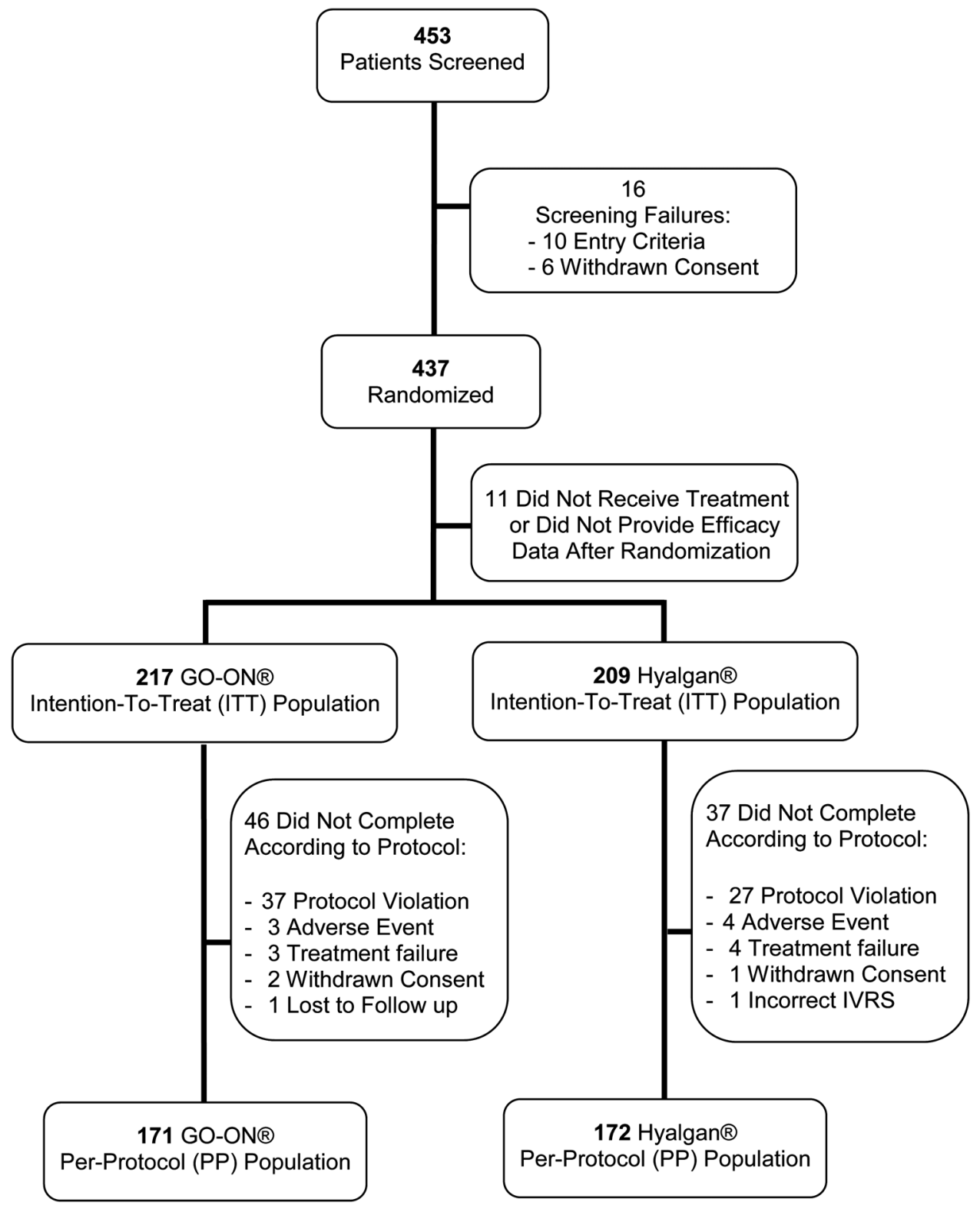

Figure 1 Trial profile.

according to a non-inferiority design, with the possibility to shift to superiority following current regulatory guidelines. ${ }^{12} 13$ The trial was registered on the European Medicines Agency database accessible at www.clinicaltrialsregister.eu (EudraCt no 2008-003875-35). Ethics approval was provided by the ethics review boards of the coordinating investigators (co-authors FB and JG) in France and Germany (Comité de Protection des Personnes, AP-HP St-Antoine Hospital, Paris and the ethics committee of Regensburg University, respectively) and by all applicable local ethics committees. After signing an informed consent, patients were screened at least 7 days before randomisation. Patients received 3-weekly injections of the test or comparator preparations (1:1 allocation ratio) and were then seen at weeks 6, 14, 20 and 26, ie, 4, 12, 18 and 24 weeks following the end of treatment.

\section{Patient selection}

Patients of either sex, aged 50-80 years, fulfilling the American College of Rheumatology clinical and radiological criteria for knee osteoarthritis, ${ }^{14}$ were enrolled if they had a history of symptoms for at least 6 months and insufficient/failed response to analgesics and/or regular non-steroidal anti-inflammatory drugs (NSAID), or were intolerant to regular NSAID or weak opioids. Current symptoms (after $\geq 2$ days wash-out from NSAID, including topical agents, or 1 day from non-narcotic analgesics) had to include global knee pain of $40 \mathrm{~mm}$ or greater on a 100 $\mathrm{mm}$ visual analogue scale (VAS), Western Ontario and McMaster Universities (WOMAC) pain subscale score of 25 or greater on the 0-100 normalised scale and Lequesne index of 4 or greater. $\mathrm{x}$-Rays (past 12 months) had to show Kellgren and Lawrence stage II or III; radiological evidence of bilateral knee osteoarthritis was accepted if global pain VAS in the contralateral knee was less than $30 \mathrm{~mm}$. Main exclusions were: isolated/predominantly patellofemoral symptomatic osteoarthritis, secondary knee osteoarthritis, symptomatic hip osteoarthritis homolateral to the target knee, inflammatory or other rheumatic diseases, clinical joint effusion, excessive $\left(\geq 8^{\circ}\right)$ varus or valgus knee deformity (at physical examination, as confirmed by standard radiograph). 
Table 1 Patient demographic and baseline characteristics in the ITT population

\begin{tabular}{lcc}
\hline & GO-ON $(\mathbf{n = 2 1 7})$ & Hyalgan $(\mathbf{n = 2 0 9})$ \\
\hline Age (years) & $67.2 \pm 7.8$ & $66.1 \pm 8.1$ \\
Women, $\mathrm{n}(\%)$ & $135(62 \%)$ & $134(64 \%)$ \\
BMI $\left(\mathrm{kg} / \mathrm{m}^{2}\right)$ & $28.0 \pm 3.0$ & $27.7 \pm 3.1$ \\
Kellgren and Lawrence, $\mathrm{n}(\%)$ & & \\
Grade II & $101(46 \%)$ & $113(54 \%)$ \\
Grade III & $116(54 \%)$ & $96(46 \%)$ \\
WOMAC index scores (0-100 scale) & & \\
Pain subscale & $47.5 \pm 14.3$ & $48.8 \pm 14.9$ \\
Stiffness subscale & $46.4 \pm 20.8$ & $48.5 \pm 19.7$ \\
Function subscale & $48.7 \pm 17.1$ & $49.3 \pm 16.4$ \\
Total index & $48.2 \pm 15.8$ & $49.1 \pm 5.3$ \\
Global knee pain VAS (0-100 mm) & $62.1 \pm 13.0$ & $64.2 \pm 13.1$ \\
Lequesne index of severity (0-24 points) & $11.2 \pm 2.9$ & $11.2 \pm 3.0$ \\
PGA VAS (0-100 mm) & $48.4 \pm 18.1$ & $47.3 \pm 17.7$ \\
ICOAP total (0-100 scale) & $46.4 \pm 16.7$ & $48.2 \pm 17.4$ \\
ICOAP constant (0-100 scale) & $43.2 \pm 18.4$ & $44.8 \pm 19.6$ \\
ICOAP intermittent (0-100 scale) & $49.2 \pm 17.0$ & $51.0 \pm 17.3$ \\
\hline
\end{tabular}

Data are mean $\pm S D$ unless otherwise indicated.

$\mathrm{BMI}$, body mass index; ICOAP, index of intermittent and constant osteoarthritis pain ITT, intention-to-treat; PGA, patient global assessment; VAS, visual analogue scale; WOMAC, Western Ontario and McMaster Universities.

\section{Treatments, randomisation, allocation concealment and blinding}

GO-ON (Rottapharm|Madaus, Monza, Italy) is a preparation of sodium hyaluronate obtained by fermentation from Streptococcus equi, with an intermediate MW (range 800000-1500000 Daltons), presented in $2.5 \mathrm{ml}$ prefilled syringes and a concentration of $10 \mathrm{mg} / \mathrm{ml}$. Hyalgan (Fidia Abano Terme, Italy) is sodium hyaluronate derived from rooster combs, with a low MW (range $500000-730000$ ) and a concentration of $10 \mathrm{mg} / \mathrm{ml}$ in $2 \mathrm{ml}$. Both preparations are recommended for cycles of 3-5-weekly injections, but studies have shown no apparent difference between the two regimens with Hyalgan. ${ }^{3}$ Treatments were therefore administered intra-articularly, using a lateral femoropatellar approach, at 3-weekly intervals.

A block randomisation list was computer-generated and electronically associated to a pack list (each pack containing one syringe) by independent groups who maintained their secrecy, having no contacts with the investigators or personnel involved in the conduct of the study. Blocks were balanced at each site and their size was secret to maintain blinding. Randomisation was centralised by an interactive voice response system: the randomisation call assigned the first available position in the randomisation list and therefore the first treatment pack (identified by a unique number) available at the site. Two additional calls were made to obtain the pack number for the second and third injections. Consequently, there was no possibility for the investigator to predict the next treatment assignment, thus ensuring adequate allocation concealment.

Given the impossibility of obtaining identical presentations of the compared medications, double-blind conditions were obtained by appointing at each site one 'injector investigator' in charge of independently administering the treatments and one 'assessor investigator' performing all assessments blinded to the treatment. Patient blinding was ensured by avoiding visual access to the injection field (eg, by a screen between the patient and his/her knee). Double-blind conditions were successfully achieved for all patients (as noted in the case record form).

Use of paracetamol up to $4 \mathrm{~g}$ per day for four consecutive days was allowed as rescue medication if unbearable pain had not improved after at least $1 \mathrm{~h}$ rest. In case of paracetamol failure or

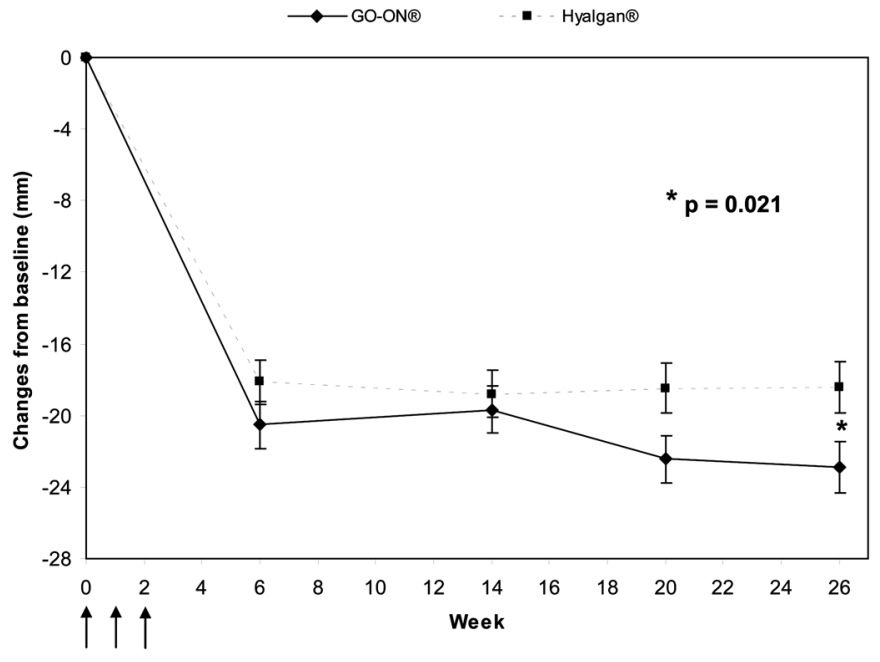

Figure 2 Intention-to-treat mean (and SE) change from baseline at each assessment time point for the Western Ontario and McMaster Universities pain subscale score in the two groups receiving GO-ON $(n=217)$ or Hyalgan $(n=209)$. The arrows indicate the intra-articular injections.

contraindication, NSAID could be prescribed for a limited and specified period. Paracetamol or NSAID had to be stopped $24 \mathrm{~h}$ or 2 days, respectively, before each assessment visit. Daily consumption of the rescue medication was recorded in a patient diary. No other use of paracetamol or NSAID (excluding aspirin $<325 \mathrm{mg} /$ day as antiaggregant) was allowed during the study, similar to opioids, intra-articular or systemic corticosteroids (washout $\geq 1$ and 3 months, respectively), or other HA preparations (washout $\geq 12$ months). Glucosamine sulphate, other glucosamines or other slow-acting drugs for osteoarthritis (eg, chondroitin sulphate, diacerhein or avocado/soybean unsaponifiables) were allowed if at a stable dosage for 3 months or more.

\section{Efficacy outcomes}

The pain subscale of the WOMAC osteoarthritis index (VAS version VA3.1) was the study primary endpoint. ${ }^{15}$ Results were normalised on a 0-100 scale for each domain, with the total index, physical function and stiffness subscales being assessed as secondary endpoints.

Other secondary efficacy endpoints included: global knee pain during the past $48 \mathrm{~h}$ on a $0-100 \mathrm{~mm}$ VAS; the Lequesne algofunctional index; ${ }^{16}$ the intermittent and constant osteoarthritis pain (ICOAP) index on the $0-100$ score transformation recommended by the OARSI and outcome measures in rheumatology (OMERACT); ${ }^{17}$ patient global assessment (PGA) on a 100 mm VAS (see supplementary material 2, available online only, for the exact wording of this and the global knee pain VAS question); the proportion of OARSI/OMERACT responders. ${ }^{18}$ The proportion of patients achieving the minimum clinically important improvement (MCII) and patient acceptable symptom state (PASS) $^{19} 20$ was also calculated for global pain VAS, WOMAC function subscale and PGA. Consumption of the rescue medication was another efficacy endpoint.

\section{Safety assessment}

The safety population comprised all patients receiving at least one injection of the study medication. Adverse events (AE) observed by the investigators or reported by the patients spontaneously or 


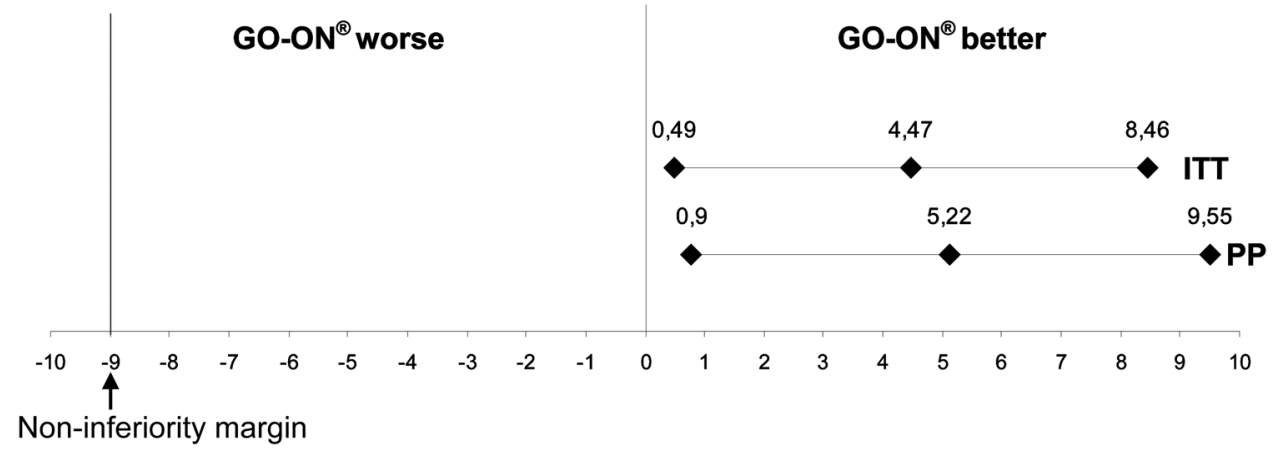

Figure 3 Point estimate and $95 \% \mathrm{Cl}$ of the difference (G0-ON vs Hyalgan, in $\mathrm{mm}$ ) in the primary outcome represented by the Western Ontario and McMaster Universities pain subscale mean improvement. Values are differences in mean changes between G0-0N and Hyalgan at 6 months after treatment in the per-protocol (PP) and intention-to-treat (ITT) populations (-9 mm was the non-inferiority margin).

following a non-leading question, were coded using the Medical Dictionary for Regulatory Activities. Particular attention was paid to local painful reactions at the injection site, postinjection reactions (eg, effusions) and acute pseudoseptic arthritis. ${ }^{21}$

\section{Statistical analysis}

The primary outcome was the difference in the mean change from baseline on the WOMAC pain subscale score at week 26 (6 months after the end of treatment). The acceptable margin for non-inferiority was preset at $-9 \mathrm{~mm}$, ie, less than the minimum perceptible clinical improvement of $-10 \mathrm{~mm} .^{22} \mathrm{GO}-\mathrm{ON}$ would be declared non-inferior to Hyalgan if the lower limit of the $95 \%$ CI lies above $-9 \mathrm{~mm}$ in both the per-protocol (PP) and intention-to-treat (ITT) populations. ${ }^{12} 13$ If the $95 \%$ CI also lies above zero in ITT, there is evidence of superiority at the $5 \%$ significance level and a two-way analysis of variance (ANOVA) with treatment and study centre as factors provided the actual $p$ value, with no multiplicity argument affecting this interpretation. ${ }^{12} 13$ The effect size was calculated according to Hedges. ${ }^{23}$

The sample size on this non-inferiority margin was calculated assuming a SD of $23 \mathrm{~mm}$, based on a previous study with Hyalgan $^{24}$ and conservatively increased by $15 \%$, resulting in 144 patients per group in the PP population to achieve a power of $90 \%$ at a significance level of $5 \%$. Assuming a $30 \%$ discontinuation rate, this was increased to 200 patients per group in the ITT population, which consisted of all randomly assigned patients with at least one injection and one post-injection assessment of the primary endpoint. ${ }^{25}$ For drop-outs and other exclusions from the PP population, missing values were replaced by the baseline value, according to the baseline observation carried-forward approach. $^{26}$

All secondary endpoints were analysed for superiority, as predetermined in the protocol. The changes in the WOMAC total index and its subscales, global knee pain, Lequesne index, PGA, ICOAP index and daily mean rescue medication consumption were analysed by ANOVA as the primary endpoint. The proportions of OARSI/OMERACT responders and patients with MCII or PASS were compared by the $\chi^{2}$ test, similar to the number of patients with $\mathrm{AE}$ and those using the rescue medication (worst case approach for drop-outs in the latter case). An analysis at week 14 (12 weeks after the end of treatment), was also performed as exploratory on all parameters. Demographic and baseline characteristics were compared by two-way ANOVA for continuous variables or the Cochran-Mantel-Haenszel test, controlling for study centre, for categorical parameters. Statistical analyses were performed using the SPSS statistical package (version 9.2).

\section{RESULTS}

Patient disposition is described in figure 1. The first patient was enrolled in November 2008 and the last patient was completed in November 2009. Out of 437 patients randomly assigned, 217 and 209 in the GO-ON and Hyalgan groups, respectively, were included in the ITT population, thus excluding only 11 patients according to the predefined criteria. Protocol violations were the main reason for exclusion from the PP population, and consisted mainly of failure to meet the inclusion/exclusion criteria or use of prohibited medications. Less than $10 \%$ of patients were withdrawn for other reasons (AE, treatment failure, withdrawal of consent, other). All withdrawals/exclusions were similarly distributed between groups.

Table 1 shows the demographic and baseline characteristics of the ITT population, without differences between groups and well representative of the common knee osteoarthritis outpatient population: mostly women (63\%), mean age approximately 67 years, moderately overweight (average body mass index approximately 28) and with Kellgren and Lawrence radiological grades II and III evenly distributed. Knee osteoarthritis symptoms were moderate to severe, with average pain and function scores in the middle of their scales, pain VAS of the signal knee greater than $60 \mathrm{~mm}$ and Lequesne index approximately 11 points. There were no differences between the ITT and PP baseline populations (data not shown).

The ITT changes from baseline in the WOMAC pain subscale are reported in figure 2. Patients in both groups improved markedly during the first month after treatment and the effect was maintained for the duration of the study, with GO-ON exhibiting an overall better trend, that was particularly consistent between 12 and 24 weeks after the end of treatment. After 6 months from the end of treatment (ie, week 26), patients who had received GO-ON had decreased their WOMAC pain score by $22.9 \pm 1.4$ $\mathrm{mm}$ (mean \pm SE), compared with $18.4 \pm 1.5 \mathrm{~mm}$ with Hyalgan in the ITT population. The primary analysis was first conducted on the PP population according to the non-inferiority study design and then on the ITT population. The changes from baseline and their $95 \%$ CI are reported in figure 3; as in both PP and ITT the lower limit of the $95 \%$ CI was higher than the predefined margin of $-9 \mathrm{~mm}$, this allowed us to conclude to the treatment noninferiority. Furthermore, as this lower limit was above 0, the ITT analysis also allowed us to conclude to the statistical superiority of GO-ON versus Hyalgan ( $\mathrm{p}=0.021$ ) (figures 2 and 3, table 2), with an effect size of 0.21 . This result did not change in a sensitivity analysis including the 11 patients excluded from the ITT population, according to a stricter ITT approach accounting for all randomly assigned patients $(p=0.026)$. 
Table 2 Mean changes (and 95\% Cl) from baseline to the endpoint (week 26, ie, 6 months after the end of treatment) in the ITT population for primary (WOMAC pain subscale) and secondary outcomes, including number (and percentages) of OARSI/OMERACT responders and of patients achieving MCII and PASS, with difference between groups and $\mathrm{p}$ value

\begin{tabular}{|c|c|c|c|c|c|}
\hline & G0-ON $(n=217)$ & Hyalgan $(n=209)$ & Difference & p Value & Effect size ${ }^{*}$ \\
\hline WOMAC pain & $-22.9(-25.7$ to -20.1$)$ & $-18.4(-21.3$ to -15.5$)$ & $-4.5(-8.5$ to -0.5$)$ & 0.021 & $0.21(0.02$ to 0.40$)$ \\
\hline WOMAC function & $-22.2(-25.1$ to -19.3$)$ & $-15.4(-18.2$ to -12.7$)$ & $-6.8(-10.7$ to -2.8$)$ & 0.0004 & $0.32(0.13$ to 0.52$)$ \\
\hline WOMAC stiffness & $-21.0(-24.3$ to -17.7$)$ & $-15.7(-19.0$ to -12.4$)$ & $-5.3(-10.0$ to -0.6$)$ & 0.027 & $0.22(0.02$ to 0.41$)$ \\
\hline WOMAC total & $-22.2(-25.0$ to -19.4$)$ & $-16.1(-18.8$ to -13.4$)$ & $-6.2(-10.0$ to -2.3$)$ & 0.001 & $0.31(0.11$ to 0.50$)$ \\
\hline VAS pain & $-33.2(-36.5$ to -29.8$)$ & $-26.7(-30.0$ to -23.4$)$ & $-6.4(-11.1$ to -1.8$)$ & 0.004 & $0.26(0.07$ to 0.45$)$ \\
\hline Lequesne index & $-4.2(-4.7$ to -3.7$)$ & $-3.0(-3.5$ to -2.5$)$ & $-1.2(-2.0$ to -0.6$)$ & 0.0002 & $0.34(0.15$ to 0.53$)$ \\
\hline ICOAP total & $-21.0(-23.8$ to -18.3$)$ & $-17.9(-20.8$ to -15.1$)$ & $-3.2(-7.0$ to 0.9$)$ & 0.13 & - \\
\hline ICOAP constant & $-21.3(-24.4$ to -18.3$)$ & $-16.9(-19.8$ to -14.0$)$ & $-4.5(-8.6$ to 0.3$)$ & 0.052 & - \\
\hline ICOAP intermittent & $-20.8(-23.6$ to -18.0$)$ & $-18.8(-21.9$ to -15.8$)$ & $-1.9(-6.0$ to 2.2$)$ & 0.32 & - \\
\hline VAS patient global & 18.8 (15.1 to 22.5$)$ & $14.3(10.6$ to 17.9$)$ & $4.6(-0.6$ to 9.8$)$ & 0.068 & - \\
\hline OARSI/OMERACT responders, n (\%) & $159(73.3 \%)$ & $122(58.4 \%)$ & $37(14.9 \%)$ & 0.001 & - \\
\hline MCII pain, n (\%) & $153(70.5 \%)$ & $122(58.4 \%)$ & $31(12.1 \%)$ & 0.009 & - \\
\hline PASS pain, $n(\%)$ & $125(57.6 \%)$ & $102(48.8 \%)$ & $23(8.8 \%)$ & 0.069 & - \\
\hline MCll function, $\mathrm{n}(\%)$ & $150(69.1 \%)$ & $117(56.0 \%)$ & $33(13.1 \%)$ & 0.005 & - \\
\hline PASS function, $\mathrm{n}(\%)$ & $137(63.1 \%)$ & $97(46.4 \%)$ & $40(16.7 \%)$ & 0.0005 & - \\
\hline MCIl patient global, n (\%) & $113(52.1 \%)$ & $92(44.0 \%)$ & $21(8.1 \%)$ & 0.096 & - \\
\hline PASS patient global, n (\%) & $126(58.1 \%)$ & $90(43.1 \%)$ & $36(15.0 \%)$ & 0.002 & - \\
\hline
\end{tabular}

All indices and scores are on a natural 0-100 mm or normalised 0-100 scale, except the Lequesne index. Baseline values are reported in table 1.

${ }^{*}$ Effect size and $95 \% \mathrm{Cl}$ shown only for continuous variables and where $\mathrm{p}<0.05$.

ICOAP, index of intermittent and constant osteoarthritis pain; ITT, intention-to-treat; MCII, minimum clinically important improvement; 0ARSI, 0steoarthritis Research Society International; OMERACT, outcome measures in rheumatology; PASS, patient acceptable symptom state; VAS, visual analogue scale; WOMAC, Western Ontario and McMaster Universities.

Table 2 shows this superiority pattern for the majority of the secondary endpoints. Global knee pain VAS decreased by over $50 \%$ with GO-ON at week 26, but less with Hyalgan (effect size 0.26). A similar degree of efficacy was detected for all WOMAC scales and the Lequesne index underwent an over 4-point decrease with GO-ON versus 3 points with Hyalgan (effect size 0.34). The degree of improvement was similar for the ICOAP index, but the difference between groups was barely significant only for constant pain, while the two preparations behaved similarly on intermittent pain. Patients had also improved their global assessment VAS by almost $20 \mathrm{~mm}$ with GO-ON, but the better trend versus Hyalgan was not significant in ITT ( $p=0.068$, table 2$)$, but only in the PP analysis $(\mathrm{p}=0.044$, data not shown).

Table 2 also shows that there were $73 \%$ OARSI/OMERACT responders 6 months after the end of treatment with GO-ON, versus $58 \%$ with Hyalgan (difference $14.9 \%, p=0.001$ ). The proportion of patients achieving MCII and PASS for global knee pain, function and PGA was also high with both treatments but significantly higher with GO-ON than with Hyalgan except for global pain PASS and MCII for PGA (table 2).

All superiority trends were similar at the 12-week endpoint (data not shown), with a significant difference in the proportion of OARSI/OMERACT responders, $69.6 \%$ with GO-ON versus $60.3 \%$ with Hyalgan $(p=0.044)$.

Patients used the rescue medication in a similar proportion: 166 out of 217 on GO-ON (77\%) and 154 out of 209 (74\%) with Hyalgan ( $\mathrm{p}=0.50)$, with a low paracetamol daily mean consumption (218 and $223 \mathrm{mg} /$ day, respectively, $\mathrm{p}=0.60)$; see supplementary text 3 (available online only).

The treatments were well tolerated. The proportion of patients reporting any $\mathrm{AE}$ in the safety population was similar: 74 out of $223(33.2 \%)$ and 75 out of $213(35.2 \%)$ with GO-ON and Hyalgan, respectively, most $\mathrm{AE}$ being unrelated to treatment. Back pain was the only AE reported with greater than $3 \%$ incidence. AE led to study discontinuation for three patients under GO-ON (worsening of knee osteoarthritis in two patients, only one of which was considered possibly related to the treatment, and one metastatic pulmonary cancer) and four patients under Hyalgan (worsening of knee osteoarthritis, post-traumatic meniscal lesion, ischaemic stroke, angiosarcoma with pleural effusion). Local $\mathrm{AE}$ are reported in table 3; both treatments were well tolerated locally. Although not statistically significant $(p=0.17)$, the proportion of patients reporting local AE was double with Hyalgan $(3.8 \%)$ compared with GO-ON (1.8\%). No case of acute pseudoseptic arthritis was observed.

\section{DISCUSSION}

In the present randomised, double-blind, controlled trial in over 400 knee osteoarthritis patients, three-weekly HA intra-articular injections decreased moderate to severe pain after 6 months by up to $50 \%$ of baseline values. Joint function improved to a similar extent and there were over $65 \%$ treatment responders on average with the two preparations used. The intermediate $\mathrm{MW}$ HA formulation GO-ON was not inferior to the reference low MW preparation Hyalgan on the WOMAC pain subscale score, but was also statistically superior on this primary and on most of the secondary outcomes as predetermined by the statistical analysis plan. ${ }^{12} 13$

While GO-ON tended to exhibit a trend for a better pattern of response throughout the study, both treatments behaved similarly well over the first month, when most of the therapeutic gain was observed, and during the first 3 months following the injection course. Afterwards, the benefit obtained with the low MW product tended to plateau, as acknowledged with most HA preparations, ${ }^{9}$ while there was a slight continuous improvement with GO-ON, ie, a more pronounced carry-over effect resulting in a statistically significant superiority on most outcomes after 6 months.

The primary endpoint was the change from baseline in the WOMAC pain subscale score, which displayed an extent of improvement similar to global knee pain VAS. The statistically significant ITT differences between treatments after 6 months were 4.5 and $6.4 \mathrm{~mm}$, respectively, ie, below the minimum perceptible clinical improvement, usually set at approximately $10 \mathrm{~mm}^{22}$ and that was used to define the non-inferiority margin 
Table 3 Number (and proportion) of patients with local AE at the injection site in the safety population

\begin{tabular}{llll}
\hline & GO-ON $(\mathbf{n = 2 2 3})$ & Hyalgan $(\mathbf{n}=\mathbf{2 1 3})$ & $\mathbf{p}$ Value \\
\hline Joint effusion/swelling & $1(0.4 \%)$ & $4(1.9 \%)$ & \\
Joint pain & $3(1.4 \%)$ & $2(0.9 \%)$ & \\
Injection site haematoma & $0(0.0 \%)$ & $2(0.9 \%)$ & \\
Injection site warmth & $0(0.0 \%)$ & $1(0.5 \%)$ & \\
$\begin{array}{l}\text { Total number of patients with } \\
\text { any of the above local AE }\end{array}$ & $4(1.8 \%)$ & $8(3.8 \%)$ & 0.17 \\
\hline
\end{tabular}

$\mathrm{AE}$, adverse event.

of $9 \mathrm{~mm}$ in this trial. Therefore, the clinical relevance of this statistical superiority is uncertain and it cannot be excluded that it is due to chance. However, the effect size ranged between 0.21 and 0.26 for the two pain endpoints, representing a small albeit clinically relevant difference. Moreover, there was a significantly higher proportion of OARSI/OMERACT responders after 6 months with GO-ON (73\%) than with Hyalgan (58\%), ie, a $15 \%$ difference that is beyond the accepted minimum clinically relevant improvement. The OARSI/OMERACT criteria require high improvement in pain or function $(\geq 50 \%$ with absolute $0-100$ change $\geq 20$ ), or a moderate improvement $(\geq 20 \%$ with $\geq 10$ absolute change) in two out of pain, function or PGA, thus representing a real clinical improvement. ${ }^{18}$ Notably, there were significantly more OARSI/OMERACT responders with GO-ON already at the 12-week endpoint, as a prelude to the better carry-over effect that was then fully substantiated after 6 months. Similar proportions and differences in response rates were observed for patients reaching the $\mathrm{MCII}^{19}$ on pain and function after 6 months. Moreover, approximately $60 \%$ of patients achieved a $\mathrm{PASS}^{20}$ with GO-ON, with a significant difference compared with Hyalgan for function, but not for pain. PGA provided a slightly lower degree of improvement, without differences between treatments in absolute terms and as MCII, but with a more than $15 \%$ significantly higher proportion of patients reaching PASS with GO-ON.

A favourable feature of the present trial is that it assessed knee osteoarthritis symptoms through several different measures, providing a complete evaluation. Beside all WOMAC subscales showing a significant advantage for the intermediate MW product, the Lequesne index resulted in an effect size of 0.36 in favour of GO-ON. In addition, pain was also assessed by the new ICOAP index; ${ }^{17}$ interestingly, patients had slightly more severe baseline values for 'intermittent' pain, in which the two treatments displayed a similar efficacy, while GO-ON was better than Hyalgan on the slightly less severe 'constant' pain.

This study also has some limitations. First, there was no placebo comparison. This might have been an issue in the case of results limited to non-inferiority, given the debated efficacy of $\mathrm{HA}$ in general. ${ }^{9}$ However, as $\mathrm{GO}-\mathrm{ON}$ was statistically superior to the low MW product and most of the differences were clinically relevant, this might represent sufficient evidence of efficacy. Moreover, ethics review boards might have raised ethical concerns in using intra-articular saline as a placebo when HA injections are now widely prescribed in knee osteoarthritis.

Second, it was not possible to provide identically appearing test and comparator preparations: the commercial preparations had to be used for obvious reasons, after appropriate packaging, and their effects may also differ given the different injected volumes. On the other hand, double-blind conditions were ensured by nominating at each site an 'injector' and a blinded 'assessor' investigator, while avoiding the patient's visual access to the injection field. Despite these difficulties, which are standard practice in these kind of trials, there was no evidence of broken double-blindness for any patient, reinforced by the strict assurance of adequately concealed random allocation by the interactive voice response system.

Third, this was a regulatory trial that was therefore industryfunded. Compared with non-industry-funded trials, sponsored studies more often yield results in support of the sponsor's drug. ${ }^{27}$ To avoid any potential allegations, an independent steering committee (FB, XC, FR, EM) supervised the trial design and study conduct, participated in blind data review meetings before database lock and provided binding recommendations for data management, finally accessing all results.

A fourth limitation was that the present trial only compared the intermediate HA product GO-ON with the reference low MW preparation, but not with a higher MW, cross-linked, formulation. While former studies suggested that high MW HA might have greater efficacy, ${ }^{4}$ in a recent meta-analysis ${ }^{11}$ showing that HA has more prolonged efficacy than intra-articular corticosteroids in knee osteoarthritis, five of the seven considered studies (and the only two high-quality trials) were performed with the low MW HA product used as reference in the present trial. Corticosteroids had a superior analgesic effect over the first 2 weeks, as expected, but from 8 weeks onwards and in particular 6 months after treatment HA had better pain control, with an effect size up to 0.39 . Another meta-analysis ${ }^{10}$ found no evidence of a clinically relevant benefit of hylan compared with lower MW HA preparations, and a recent trial concluded to the non-inferiority between another intermediate MW HA and hylan G-F20.28 Actually, a study of single intra-articular hylan injection showed only a modest, albeit significant, benefit versus placebo over 6 months on knee pain, with less OARSI/ OMERACT responders than with GO-ON in the present trial and a non-significant difference with placebo. ${ }^{29}$

A recent study has shown that low MW (50 000 Daltons) HA may have pro-inflammatory activity on chondrocytes, while intermediate MW preparations were neutral in this model of inflammation. ${ }^{30}$ It is difficult to transpose in-vitro results to humans and to the actual preparations used here, but this may partly explain the efficacy results of the present study, with good safety at the injection site compared with even higher MW products. Indeed, while in a recent trial hylan showed only a trend towards a higher incidence of local adverse reactions compared with placebo, ${ }^{29}$ previous studies suggested a doubling risk of local AE with the high MW HA preparation compared with lower MW products, ${ }^{10}$ which may be due to peptide contaminants, formaldehyde, or crystal-induced inflammation. In the present study, GO-ON and Hyalgan were equally well tolerated at the injection site, although numerically more local reactions occurred with Hyalgan than with GO-ON, therefore showing a very good safety of the latter.

In conclusion, this trial shows that the intermediate MW HA preparation GO-ON is effective on knee osteoarthritis symptoms over 6 months after a 3-weekly injection course, and may be more effective than the reference low MW formulation. Further studies are warranted to elucidate the mechanism of this possible superiority and more pronounced carry-over effect and to test whether this selected HA preparation has a prominent therapeutic profile compared with other HA products.

Acknowledgements The authors wish to thank all the clinical investigators who took part in the conduct of this trial and provided and cared for study patients.

Funding This was a trial conducted for regulatory purposes and therefore received full financial support from the sponsor Rottapharm |Madaus. Co-authors SC, MD, GG and LCR are scientists from the Department of Clinical Pharmacology of 
Rottapharm | Madaus and agreed individually to be listed as co-authors due to their involvement in the study; there was no other involvement of the sponsor as a corporate entity in the conduct of the study and in the manuscript submission process. Co-authors FB and JG were the study principal investigators and received research grants for this. Co-authors FB, XC, FR and EM received a reimbursement fee for their involvement in the steering committee of the study.

Competing interests Rottapharm |Madaus is the sponsor of the study and the manufacturer of one of the preparations tested in this study (GO-ON) and has this preparation on the market in different countries of the world. The full trial protocol, all study documents including completed case record forms and the complete statistical database are available from the sponsor's good clinical practice archives.

Ethics approval Ethics approval was provided by the Comité de Protection des Personnes, AP-HP St-Antoine Hospital, Paris and the ethics committee of Regensburg University and by all applicable local ethics committees.

Patient consent Obtained.

Provenance and peer review Not commissioned; externally peer reviewed.

\section{REFERENCES}

1. Balazs EA, Denlinger JL. Viscosupplementation: a new concept in the treatment of osteoarthritis. J Rheumatol Supp/ 1993;39:3-9.

2. Wang CT, Lin J, Chang CJ, et al. Therapeutic effects of hyaluronic acid on osteoarthritis of the knee. A meta-analysis of randomized controlled trials. J Bone Joint Surg Am 2004;86-A:538-45.

3. Bellamy N, Campbell J, Robinson V, et al. Intraarticular corticosteroid for treatment of osteoarthritis of the knee. Cochrane Database Syst Rev 2006;(2):CD005321.

4. Lo GH, LaValley M, McAlindon T, et al. Intra-articular hyaluronic acid in treatment of knee osteoarthritis: a meta-analysis. JAMA 2003;290:3115-21.

5. Modawal A, Ferrer M, Choi HK, et al. Hyaluronic acid injections relieve knee pain. $J$ Fam Pract 2005:54:758-67.

6. Arrich J, Piribauer F, Mad P, et al. Intra-articular hyaluronic acid for the treatment of osteoarthritis of the knee: systematic review and meta-analysis. Can Med Assoc J 2005; 172:1039-43.

7. Medina JM, Thomas A, Denegar CR. Knee osteoarthritis: should your patient opt for hyaluronic acid injection? J Fam Pract 2006;55:669-75.

8. Zhang W, Moskowitz RW, Nuki G, et al. OARSI recommendations for the management of hip and knee osteoarthritis. Part II: OARSI evidence-based, expert consensus guidelines. Osteoarthr Cartil 2008;16:137-62.

9 Zhang W, Nuki G, Moskowitz RW. OARSI recommendations for the management of hip and knee osteoarthritis part III: changes in evidence following systematic cumulative update of research published through January 2009. Osteoarthr Cartilage 2010;18:476-99.

10. Reichenbach S, Blank S, Rutjes AW, et al. Hylan versus hyaluronic acid for osteoarthritis of the knee: a systematic review and meta-analysis. Arthritis Rheum 2007:57:1410-18.

11. Bannuru RR, Natov NS, Obadan IE, et al. Therapeutic trajectory of hyaluronic acid versus corticosteroids in the treatment of knee osteoarthritis: a systematic review and meta-analysis. Arthritis Rheum 2009;61:1704-11.

12. Committee for Proprietary Medicinal Products. Points to consider on switching between superiority and non-inferiority (CPMP/EWP/482/99). London: European Medicines Agency, 2000

13. Center for Drug Evaluation and Research. Guidance for Industry: Non-Inferiority Clinical Trials (Draft guidance). Silver Spring, MD: Food and Drug Administration, 2010.
14. Altman R, Asch E, Bloch D, et al. Development of criteria for the classification and reporting of osteoarthritis. Classification of osteoarthritis of the knee. Diagnostic and Therapeutic Criteria Committee of the American Rheumatism Association. Arthritis Rheum 1986;29:1039-49.

15. Bellamy N, Buchanan WW, Goldsmith CH, et al. Validation study of WOMAC: a health status instrument for measuring clinically important patient relevant outcomes to antirheumatic drug therapy in patients with osteoarthritis of the hip or knee. J Rheumatol 1988;15:1833-40.

16. Lequesne MG, Mery C, Samson M, et al. Indexes of severity for osteoarthritis of the hip and knee. Validation-value in comparison with other assessment tests. Scand J Rheumatol Supp/ 1987;65:85-9.

17 Hawker GA, Davis AM, French MR, et al. Development and preliminary psychometric testing of a new OA pain measure - an OARSI/OMERACT initiative. Osteoarthr Cartilage 2008;16:409-14.

18. Pham T, van der Heijde D, Altman RD, et al. OMERACT-OARSI initiative: Osteoarthritis Research Society International set of responder criteria for osteoarthritis clinical trials revisited. Osteoarthr Cartil 2004;12:389-99.

19. Tubach F, Ravaud P, Baron G, et al. Evaluation of clinically relevant changes in patient reported outcomes in knee and hip osteoarthritis: the minimal clinically important improvement. Ann Rheum Dis 2005;64:29-33.

20. Tubach F, Ravaud P, Baron G, et al. Evaluation of clinically relevant states in patient reported outcomes in knee and hip osteoarthritis: the patient acceptable symptom state. Ann Rheum Dis 2005;64:34-7.

21 Maheu E, Bonvarlet JP and the Paris Rheumatologists Association. Acute pseudoseptic arthritis post hyaluronane $(\mathrm{HA})$ intra-articular injections. Results of a French survey in rheumatology practice (Abstract). Ann Rheum Dis 2003;62:268

22. Ehrich EW, Davies GM, Watson DJ, et al. Minimal perceptible clinical improvement with the Western Ontario and McMaster Universities osteoarthritis index questionnaire and global assessments in patients with osteoarthritis. J Rheumatol 2000;27:2635-41

23. Hedges LV, Olkin I. Statistical methods for meta-analysis. Orlando, FL: Academic Press, 1985

24. Thompson JI, Huang YW, Zaibel R. Safety and efficacy of fermentationderived high molecular weight sodium hyaluronate - a clinical study in patients with osteoarthritis of the knee (Abstract). Osteoarthr Cartilage 2002;10 (Suppl 1):S70-1.

25. Committee for Proprietary Medicinal Products. Note for guidance on statistical principles for clinical trials (CPMP/ICH/363/96). London: European Medicines Agency, 1998.

26. Committee for Medicinal Products for Human Use. Guideline on missing data in confirmatory clinical trials (CPMP/EWP/1776/99 Rev.1). London: European Medicines Agency, 2011

27. Bekelman JE, Li Y, Gross CP. Scope and impact of financial conflicts of interest in biomedical research: a systematic review. JAMA 2003;289:454-65.

28. Maheu E, Zaim M, Appelboom T, et al. Comparative efficacy and safety of two different molecular weight (MW) hyaluronans F60027 and Hylan G-F20 in symptomatic osteoarthritis of the knee (KOA). Results of a non-inferiority, prospective randomized, controlled trial. Clin Exp Rheumatol 2011;29:527-35.

29. Chevalier $\mathbf{X}$, Jerosch J, Goupille P, et al. Single, intra-articular treatment with 6 $\mathrm{ml}$ hylan G-F 20 in patients with symptomatic primary osteoarthritis of the knee: a randomised, multicentre, double-blind, placebo controlled trial. Ann Rheum Dis 2010;69:113-19.

30. Campo GM, Avenoso A, Campo S, et al. Differential effect of molecular mass hyaluronan on lipopolysaccharide-induced damage in chondrocytes. Innate Immun 2010:16:48-63. 